\title{
Elektronische Simulation der Reizausbreitung im Herzen
}

\author{
H. Müller, J. Nagel, M. Schaldach \\ Zentralinstitut für Biomedizinische Technik, Universität Erlangen-Nürnberg
}

Die moderne Elektronik erlaubt es, komplizierte Abläufe zu simulieren und durch geeignete Ausgabegeräte in einer leicht verständlichen Form darzustellen. Das vorliegende Gerät simuliert die Reizausbreitung im menschlichen Herzen und die Darstellung des zugehörigen EKGs auf einem Farbfernsehgerät. Folgende Programme lassen sich über Funktionstasten abrufen:

$1=$ Normales Herz

$2=2: 1$ Block

$3=3: 1$ Block

4 = Totaler Block

5 = Tachykardie

6 = Bradykardie

- Frequenz der Sinuserregung digital einstellbar

- Reizschwelle des Herzens digital einstellbar

- Oberlagerung des EKGs mit Rauschen zur Demonstration des Schrittmacherverhaltens bei gestörtem EKG

- EKG-Amplitude kann verkleinert werden um Vergrößerung des Obergangwiderstands Herz - Elektrode zu simulieren

- Batteriestrom des Schrittmachers kann digital angezeigt werden

- Reizausbreitung in Zeitlupe, da Darstellung in Echtzeit vom Auge nicht genügend aufgelöst wird.

Abb. 1 zeigt ein Schirmbildfoto des Gerätes bei eingestelltem 2:1 Block mit Schrittmacher, dessen Impulse als senkrechte Striche im EKG dargestellt sind. Aus den unterschiedlichen Färbungen der Reizleitung ist zu entnehmen, welche Teile des Herzens sich in Erregung befinden und welche nicht, wobei der Bezug zum EKG durch die gestrichelte Linie unterhalb des Herzens hergestellt wird.

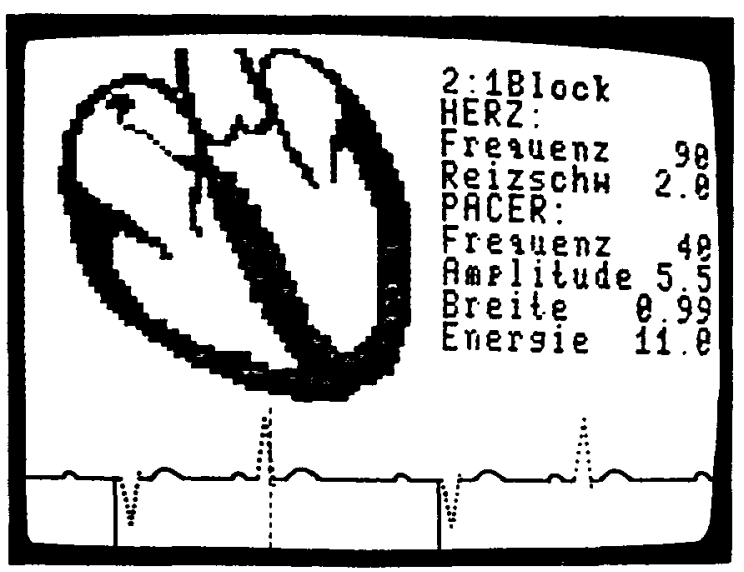

Abb. 2 zeigt das Blockschaitbild des Gerätes. Ober eine Funktions- und numerische Tastatur können verschiedene Programme angewählt und einige herzspezifische Werte sowie Sonderfunktionen eingegeben werden. Bei verschiedenen Reizbildungs- und Reizleitungsstörungen kann die Wirksamkeit eines Schrittmachers demonstriert werden. Ein Interface für den AnschluB eines handelsublichen Gerätes ist im Gerät implementiert. Ein 8-Bit Mikroprozessor übernimmt sowohl die zur Simulation und zur Darstellung der Reizausbreitung sowie des EKGs erforderlichen Berechnungen, als auch die Parameterbestimung der vom Schrittmacher abgegebenen Impulse. 
Die Daten der Herzsilhouette mit eingeblendeter Reizleitung werden in einem Bildwiederholspeicher abgelegt, da der Mikroprozessor zeitlich nicht in der Lage ist ein Videosignal zu erzeugen. Mit Hilfe eines Zeichengenerators werden alle fur den Bildaufbau nötigen grafischen und alphanumerischen Zeichen generiert. Eine punktweise Darstellung ist hierdurch jedoch nicht möglich, weshalb zur Ausgabe des EKGs ein separater Bildwiederholspeicher notwendig ist. Im nachfolgenden Video - Mischer werden die erzeugten Signale gemischt und mit den vom Taktgenerator erzeugten Synchronsignalen versehen. ober einen HF - Modulator wird das Signal in die Antennenbuchse eines normalen Farbfernsehgerätes eingespeist und als Bild ausgegeben. handen ist und wenn $\mathbf{j a}$, wird die Frequenz berechnet sowie die Amplitude und Pulsbreite gemessen und angezeigt. Hieran schließt sich die Berechnung eines neuen EKG-Punktes in Abhängigkeit des gewählten Programms und der Parameter des Schrittmachers an; die Ablage der Information erfolgt wiederum im EKG-Bildwiederholspeicher. Startet ein neuer QRS-Komplex, so wird die Schlagfrequenz des Herzens ermittelt und angezeigt. Die Farbänderung der Reizleitungstrakte im Bild erfolgt unter Bezugnahme des dargestellten EKGs an einem unter dem Herzen befindlichen Cursor. Dabei werden sowohl Eigenerregungen als auch vom Schrittmacher stimulierte Erregungen beriucksichtigt. Hieran schließt sich die Abfrage der Tastatur mit der Anzeige und Verarbeitung eventuell

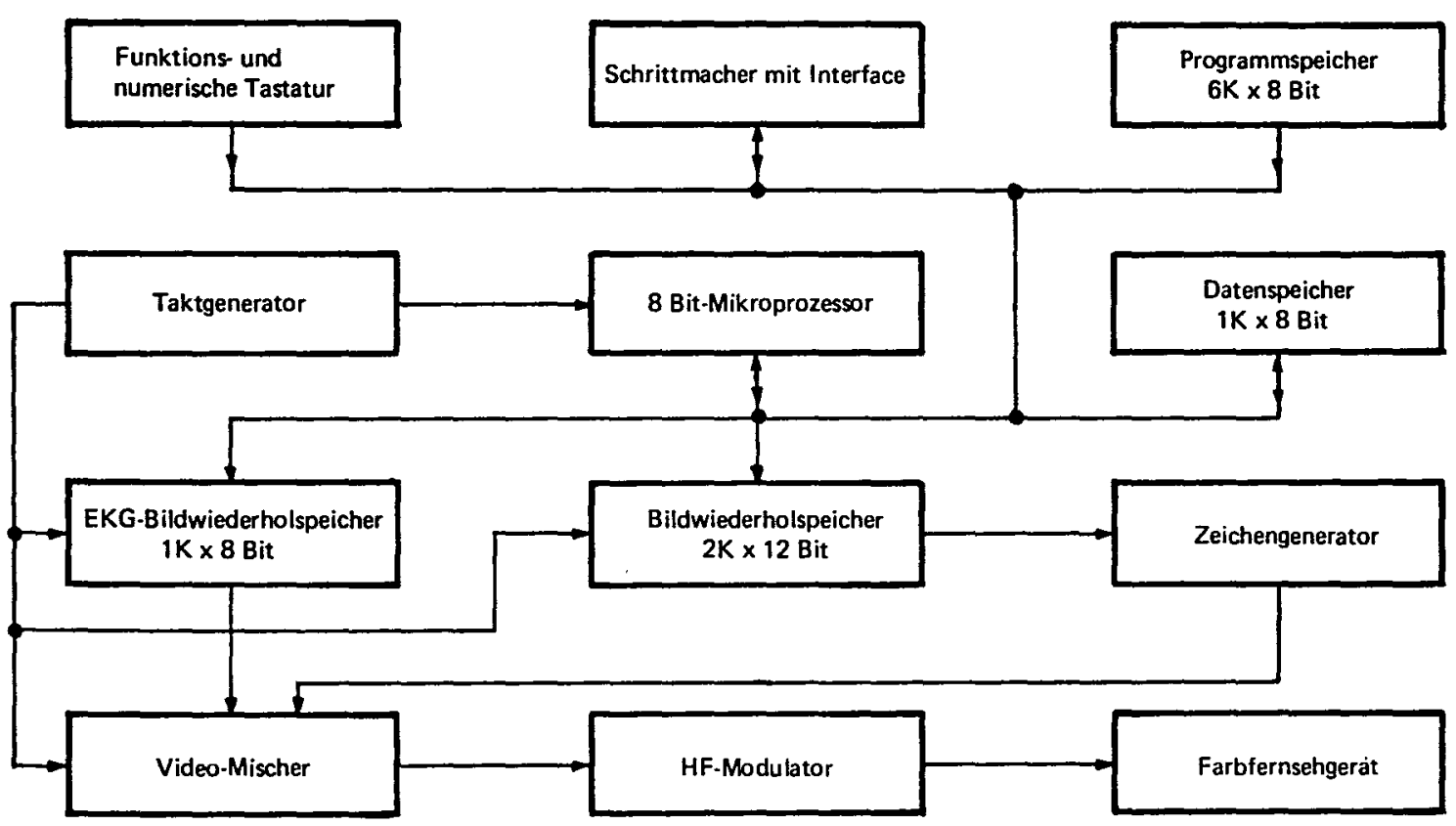

Abb. 2 Blockschaltbild

Das FluBdiagramm ist in Abb. 3 dargestellt. Nach dem Starten des Mikroprozessors wird eine Obersicht über die verfügbaren Programe angezeigt. Nachdem uber die Tastatur ein Programm gewählt wurde, wird die Herzsilhouette auf den Bildschirm ausgegeben und das Hauptprogram gestartet. Die folgende Programschleife wird alle $5 \mathrm{~ms}$ durchlaufen. Es wird jedesmal abgefragt ob ein Schrittmacherimpuls vor- eingegebener Werte an. Danach springt das Programm an den Anfang der $5 \mathrm{~ms}$ Schleife zurück und beginnt einen neuen Zyklus. Bei Betätigung der Obersichtstaste listet der Mikroprozessor die wählbaren Programe auf und wartet auf eine neue Eingabe.

Das beschriebene Gerät erweist sich als wertvolles Hilfsmittel zur Veranschaulichung der Reizaus- 
breitung im Herzen im Zusammenhang mit den meBbaren Potentialen. Durch AnschluB eines Schrittmachers kann dessen Verhalten bei verschiedenen Rhythmusstörungen des Herzens sowie bei Störfällen beobachtet werden.

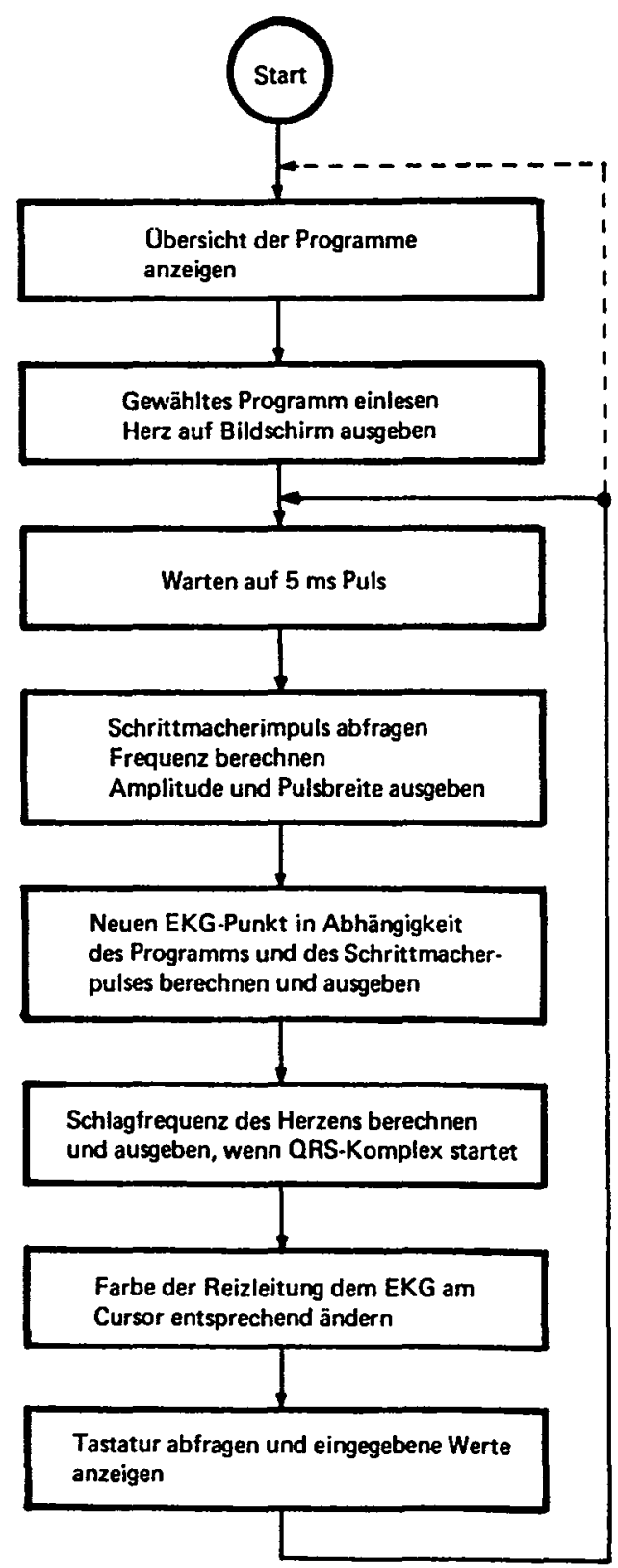

Abb. 3 Flußdiagramm

\section{Literatur:}

(1) Heinecker, R. EKG-Fibel

Georg Thieme Verlag (Stuttgart 1973)

(2) Lepeschkin, E. Das Elektrokardiogramm Verlag von Theodor Steinkopff (Dresden Leipzig 1957)

(3) Netter, F. Farbatlanten der Medizin Band 1: Herz Georg Thieme Verlag (Stuttgart 1976)

(4) 2650 Registered Microprocessor Manual Set Signetics Corporation (Sunnyvale, California 1975)

(5) Valvo Brief 3. April 1978

Mikroprozessor-gesteuerte Bildschirmspiele (PVF 2636-System und RAM-BildwiederholspeicherSystem)

Valvo Hamburg 